\title{
Painful ophthalmoplegia with normal cranial imaging
}

\author{
Chih-Hsien Hung ${ }^{1,2+}$, Kuo-Hsuan Chang ${ }^{3 \dagger}$, Chun-Che Chu ${ }^{3}$, Ming-Feng Liao ${ }^{3}$, Hong-Shiu Chang ${ }^{3}$, Rong-Kuo Lyu ${ }^{3}$, \\ Yi-Ming Wu ${ }^{4}$, Yao-Liang Chen ${ }^{5}$, Chiou-Lian Lai ${ }^{2}$, Hsiao-Jung Tseng ${ }^{6}$ and Long-Sun Ro ${ }^{3^{*}}$
}

\begin{abstract}
Background: Painful ophthalmoplegia with normal cranial imaging is rare and confined to limited etiologies. In this study, we aimed to elucidate these causes by evaluating clinical presentations and treatment responses.

Methods: Cases of painful ophthalmoplegia with normal cranial MRI at a single center between January 2001 and June 2011 were retrospectively reviewed. Diagnoses of painful ophthalmoplegia were made according to the recommendations of the International Headache Society.

Results: Of the 58 painful ophthalmoplegia cases (53 patients), 26 (44.8\%) were diagnosed as ocular diabetic neuropathy, 27 (46.6\%) as benign Tolosa-Hunt syndrome (THS), and 5 (8.6\%) as ophthalmoplegic migraine (OM). Patients with ocular diabetic neuropathy were significantly older ( $62.8 \pm 7.8$ years) than those with benign THS (56.3 \pm 12.0 years) or OM ( $45.8 \pm 23.0$ years) $(p<0.05)$. Cranial nerve involvement was similar among groups. Pupil sparing was dominant in each group. Patients with benign THS and OM responded exquisitely to glucocorticoid treatment with resolved diplopia, whereas patients with ocular diabetic neuropathy didn't $(p<0.05)$. Patients with OM recovered more rapidly than the other groups did $(p<0.05)$. Overall, most patients $(94.8 \%)$ recovered completely during the follow-up period.
\end{abstract}

Conclusions: Ocular diabetic neuropathy and benign THS accounted for most of the painful ophthalmoplegias in patients with normal cranial imaging. Patient outcomes were generally good.

Keywords: Headache, Ocular diabetic neuropathy, Ophthalmoplegic migraine, Painful ophthalmoplegia, Tolosa-Hunt syndrome

\section{Background}

Painful ophthalmoplegia consists of periorbital or hemicranial pain with ipsilateral ocular motor nerve palsies [1]. The syndrome involves diverse causes; therefore, a comprehensive evaluation is essential. Contrast-enhanced magnetic resonance imaging (MRI) provides sensitive detection of structural abnormalities, such as trauma, infection, malignancy, or vascular anomaly $[2,3]$.

However, neuroimaging studies frequently yield negative findings in patients with painful ophthalmoplegia [4]. Cranial neuropathies in diabetic patients due to

\footnotetext{
*Correspondence: cgrols@adm.cgmh.org.tw

tEqual contributors

${ }^{3}$ Department of Neurology, Linkou Campus, Chang Gung Memorial Hospital and University College of Medicine, Gueishan Township, Taiwan
}

Full list of author information is available at the end of the article microvasculopathy occasionally demonstrate painful ophthalmoplegia with normal neuroimaging studies [5,6]. Maintaining optimal glycemic control may aid recovery [5]. Ophthalmoplegic migraine (OM), which frequently occurs in childhood [7], may also present as painful ophthalmoplegia in adulthood upon normal imaging studies [8]. The use of glucocorticoids along with migraine prophylaxes, such as $\beta$-blockers or calcium channel blockers, may hasten recovery [8]. Occasionally, idiopathic inflammation of the cavernous sinus (CS) can cause painful ophthalmoplegia. Tolosa-Hunt syndrome (THS) is caused by an inflammatory process of unknown etiology. Although granulomatous tissues in the CS can often be identified in these patients (referred to as inflammatory THS), half of the patients with clinical presentation and diagnosis of THS have no radiographic evidence of inflammation $[9,10]$. The eponym "benign THS" has been introduced

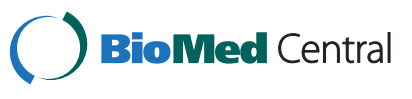


for patients with THS and normal neuroimaging findings [9]. Glucocorticoids are considered as standard treatment for patients with THS $[1,10]$.

Given that the choice of treatment depends on an accurate diagnosis, it is essential to understand the clinical presentations in patients with MRI-negative painful ophthalmoplegia. In this study, we aimed to elucidate the causes in this group of patients by evaluating their clinical presentations and responses to treatment. These results will be important for guiding appropriate treatment decisions in patients with painful ophthalmoplegia.

\section{Methods}

Consecutive adult patients referred with painful ophthalmoplegia to Chang Gung Memorial Hospital were collected from January 2001 to June 2011. Their in-hospital medical chart records were retrospectively reviewed with approval of the Institutional Review Board of the Chang Gung Memorial Hospital (license ID: 100-0136B). Patients who developed periorbital or hemicranial pain combined with ipsilateral oculomotor, trochlear, and/or abducent palsies were recruited in this study. All patients had appropriate laboratory workup, including complete hematologic and routine tests, electrolytes, renal and liver function test, anti-nuclear antibody, thyroid function, C-reactive protein, elevated sedimentation rate (ESR), fasting glucose, and glycohemoglobin (HbA1c). All recruited patients also underwent cranial MRI and cerebral vascular investigations (MR angiography or digital subtraction angiography). None of the patients had concurrent infection, malignancy, Sjögren's syndrome, systemic lupus erythematosus, rheumatoid arthritis, vasculitis, cranial herpes zoster, Horton arteritis, or immunocompromised status.

The diagnosis of painful ophthalmoplegia was made according to the recommendations of the International Headache Society (IHS), $2^{\text {nd }}$ edition (ICDH-2) [4]. Patients with diabetes (fasting plasma glucose $\geq 126 \mathrm{mg} / \mathrm{dL}$ or HbA1c $\geq 6.5 \%)$ developing painful ophthalmoplegia without other attribution after workup were designated as ocular diabetic neuropathy $[4,11]$. Patients with idiopathic unilateral painful ophthalmoplegia fulfilling the IHS criteria for THS and with normal cranial imaging were designated as benign THS [9]. Since THS symptoms may resolve spontaneously when left untreated $[1,10,12]$, patients with idiopathic painful ophthalmoplegia fulfilled THS criteria (A, B and C) and self-remission within 3 months were also considered as those with THS. These patients with THS may have delayed diplopia improvement after 6-8 weeks of glucocorticoid treatment [13]; therefore, patients fulfilling the THS criteria with pain resolving within 72 hours after treatment, but without immediate improvement in their diplopia, were also allocated to the THS group. Patients with migrainous headache accompanied or followed by ophthalmoplegia within 4 days in accordance with the IHS criteria for OM was allocated to the OM group [4]. Patients with a single attack fulfilling the OM criteria were also considered as having $\mathrm{OM}[8,14]$. All enrolled patients underwent a follow-up period of at least 6 months to ensure no other etiology was identified.

All enrolled patients underwent cranial MRI with a standard head coil on a 1.5- to 3-T MR scanner. The predefined MR scan parameters used in this study were as follows: $\mathrm{TR}=450-550 \mathrm{~ms}$, $\mathrm{TE}=9.9-12 \mathrm{~ms}$, field of view $(\mathrm{FOV})=200 \times 200 \mathrm{~mm}$, and matrix $=512 \times 512$ in transverse and coronal T1-weighted spin-echo sequences before and after intravenous administration of gadolinium enhancement $(0.2 \mathrm{ml} / \mathrm{kg})$; TR $=3114-4000 \mathrm{~ms}, \mathrm{TE}=81-$ $90 \mathrm{~ms}$, FOV $=200 \times 200 \mathrm{~mm}$, matrix $=512 \times 512$ in trans verse T2 fast spin-echo sequence; and TR $=6000-9000$, $\mathrm{TE}=105-142 \mathrm{~ms}$, FOV $=200 \times 200 \mathrm{~mm}$, and matrix $=$ $512 \times 512$ for transverse fluid-attenuated inversion recovery sequence (FLAIR). Sections were either 3 or $5 \mathrm{~mm}$ in thickness. Cerebral vascular investigations, including MR angiography or digital subtraction angiography, were performed in all recruited patients to exclude vascular anomalies.

Cranial scans of painful ophthalmoplegias were individually and retrospectively reviewed, by 2 experienced neuroradiologists ( $\mathrm{Wu}, \mathrm{YM}$ and $\mathrm{Chen}, \mathrm{YL}$ ) with a focus on the parasellar area and orbits. Both reviewers were aware of the symptom of painful ophthalmoplegia but were blind to the final diagnosis. Only cases with normal neuroimaging findings, consented by both reviewers, were further analyzed.

Glucocorticoid dosages and response to treatment were registered. A positive response to glucocorticoids was defined as relief of symptoms or improved neurologic signs within 3 days of glucocorticoid treatment initiation $[4,15,16]$.

Statistical analyses were performed using SPSS statistical software (version 17.0, Chicago, IL, USA). Descriptive statistics were expressed mean \pm standard deviation (SD) for continuous data, whereas the number with its percentage for categorical data. Continuous variables were compared using Kruskal Wallis tests followed by post-hoc Dunn tests if statistically significant. Categorical variables were compared using chi-square test. Disease course was estimated according to the Kaplan-Meier method. The endpoint of the primary study was the time until complete resolution of symptoms and signs. All $\mathrm{p}$ values were twotailed. In all tests, $\mathrm{p}<0.05$ was considered statistically significant.

\section{Results}

There were 121 referrals with symptomatic diagnoses of unilateral painful ophthalmoplegia (Figure 1). Four patients were excluded because of incomplete neuroimaging 


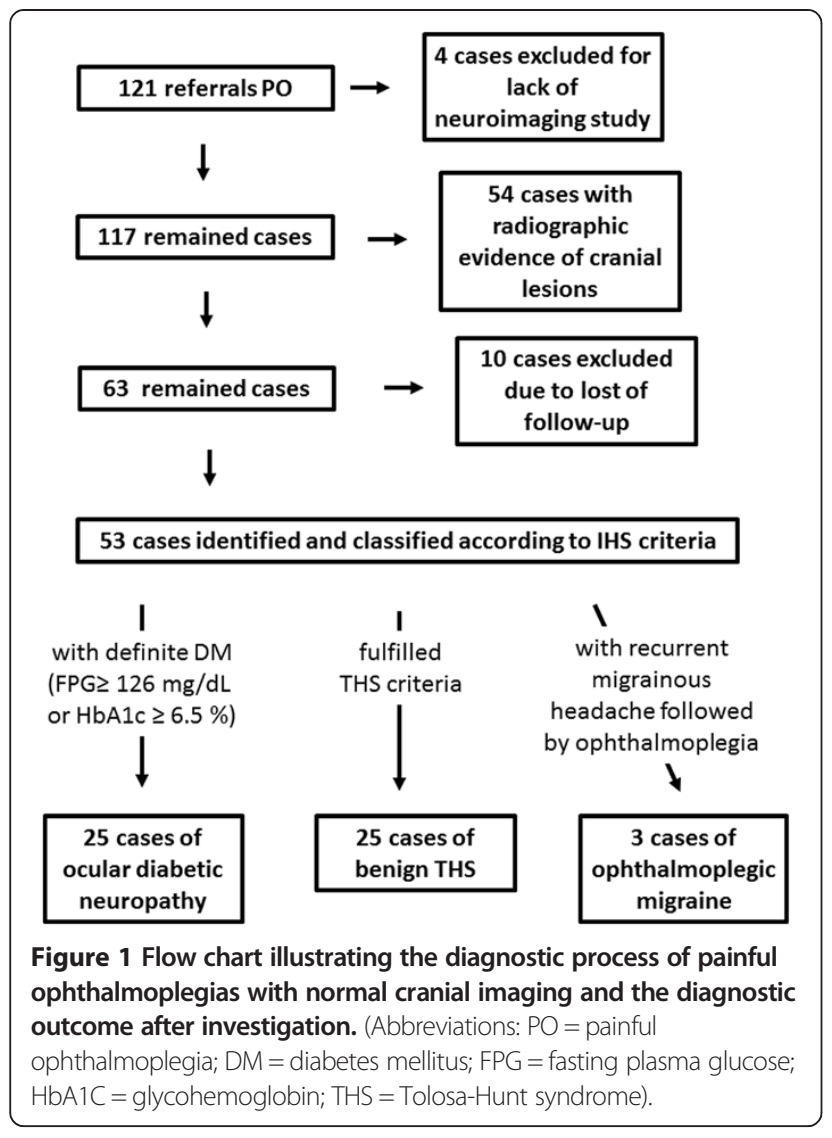

and/or cerebral vascular investigations. Of the remaining 117 patients, 54 patients with radiographic evidence of cranial lesions were excluded because of the following conditions: carotid-cavernous fistula (10 patients), P-com aneurysm (2 patients), primary head and neck neoplasms (6 patients), primary intracranial neoplasm (3 patients), cavernous sinus metastases (2 patients), sinonasal infection (5 patients), orbital infection (4 patients), trauma (1 patient), and idiopathic inflammatory Tolosa-Hunt syndrome (21 patients). Ten patients with initial normal neuroimaging were also excluded because of the lack of follow-up. Finally, 53 qualified patients with normal neuroimaging data were recruited for this study. Twenty-five patients (47.2\%) were diagnosed as having ocular diabetic neuropathy, and $25(47.2 \%)$ and $3(5.7 \%)$ patients were diagnosed with benign THS and OM, respectively (Table 1 ). Recurrent episodes were reported in 1 patient (4.0\%) with ocular diabetic neuropathy, 1 patient (33\%) with OM, and 2 patients $(8.0 \%)$ with benign THS. A total of 58 episodes were recruited for further analysis.

Demographic data and clinical presentations between the groups are summarized in Table 1. Patients with ocular diabetic neuropathy were significantly older than patients with benign THS (post-hoc tests; $p=0.045$ ) and patients with OM $(p=0.004)$ (Table 1). All patients with diabetic ophthalmoplegia had type II diabetes mellitus (DM), and the known duration of diabetes was $9.9 \pm 9.4$ years. Five patients $(20.0 \%)$ were newly diagnosed with DM. No patient with final diagnosis of THS or OM had diabetes or impaired fasting glucose. All patients with OM had adultonset migrainous headache.

Cranial nerve involvement was not significantly different among groups (Table 1). The most frequently involved cranial nerve was the oculomotor nerve in all groups (74.1\%), followed by the trochlear nerve (53.4\%) and the abducens nerve (39.7\%). Pupillary function was not altered in most patients; pupil sparing was common in patients with ocular diabetic neuropathy as well as in other groups. Compared to patients with diabetic ophthalmoplegia, those with benign THS and OM were prone to multiple ocular motor neuropathies, even though there was no significant difference among groups.

In the laboratory examinations, the ESR was not significantly different within groups. Anti-nuclear antibody (ANA) and rheumatoid factor (RF) were also non-specific. Compared to benign THS $(5.9 \pm 2.5 \%)$ and OM $(6.2 \pm$ $0.6 \%)$, ocular diabetic neuropathy was associated with higher levels of glycohemoglobin $(8.4 \pm 1.8 \%, p=0.003)$.

All enrolled patients underwent a follow-up period of at least 6 months to ensure no other etiology was identified. The mean duration from initial symptoms to the last follow-up was 3.4, 4.9, and 3.6 years in patients with ocular diabetic neuropathy, benign THS, and OM, respectively. No patients had newly diagnosed autoimmune disease, intracranial malignancy, vascular malformation, sarcoidosis, central nervous system (CNS) infection, or HIV infection during the follow-up period.

The duration from onset to complete recovery in ocular diabetic neuropathy was $2.0 \pm 1.0$ months, which was similar to that in benign THS (1.6 \pm 0.8 months). Recovery was significantly more rapid $(0.9 \pm 0.4$ months $)$ in OM than either in benign THS or in ocular diabetic neuropathy (log-rank test, $p=0.005$, Figure 2). In most cases $(\mathrm{n}=55,94.8 \%)$, patients reported complete recovery within 3 months, except for 1 case of benign THS (3.7\%) and 2 cases of ocular diabetic neuropathy $(8.3 \%)$ with minor sequelae of diplopia.

Glucocorticoids were prescribed in 24 episodes (88.9\%) of benign THS and 4 of OM (80.0\%). Therapeutic trial of glucocorticoids was used in 13 episodes (50.0\%) of ocular diabetic neuropathy because of initially inconclusive diagnoses. Four patients with benign THS and 2 patients with ocular diabetic neuropathy improved before glucocorticoid use and, thus, were excluded from these analyses. The mean dose of prednisolone was $1.3 \pm 3.7 \mathrm{mg} / \mathrm{kg} /$ day.

Immediate pain relief within 72 hours of treatment was observed in nearly all patients. Regarding resolved diplopia, patients with benign THS and OM responded well to glucocorticoids ( $95.0 \%$ and $100.0 \%$, respectively), but only 
Table 1 Demographic data and clinical manifestations of painful ophthalmoplegia with normal neuroimaging

\begin{tabular}{|c|c|c|c|c|c|}
\hline & Ocular diabetic neuropathy & Benign Tolosa-Hunt syndrome & Ophthalmoplegic migraine & Total & $p$ value \\
\hline $\mathrm{N}(\%)$ & $25(47.2)$ & $25(47.2)$ & $3(5.7)$ & 53 & \\
\hline $\mathrm{N}$ of episodes & 26 & 27 & 5 & 58 & \\
\hline$M / F$ & $12 / 13$ & $10 / 15$ & $3 / 0$ & $25 / 28$ & \\
\hline Age (year) & $62.8 \pm 7.8$ & $56.3 \pm 12.0$ & $45.8 \pm 23.0$ & $58.3 \pm 12.4$ & $0.008^{*}$ \\
\hline \multicolumn{6}{|c|}{ Involvement of cranial nerves, $\mathrm{N}(\%)$} \\
\hline CN3 & $19(73.1)$ & $20(74.1)$ & $4(80.0)$ & $43(74.1)$ & 0.949 \\
\hline CN4 & $9(34.6)$ & $15(55.6)$ & $2(40.0)$ & $23(39.7)$ & 0.301 \\
\hline CN6 & $13(50.0)$ & $16(59.3)$ & $2(40.0)$ & $31(53.4)$ & 0.652 \\
\hline Ptosis & $16(61.5)$ & $11(40.7)$ & $1(20.0)$ & $28(48.3)$ & 0.132 \\
\hline Pupillary dysfunction & $7(26.9)$ & $6(22.2)$ & $0(0.0)$ & $13(22.4)$ & 0.417 \\
\hline Multiple CNs involvement & $10(38.5)$ & $18(66.7)$ & $3(60.0)$ & $31(53.4)$ & 0.115 \\
\hline \multicolumn{6}{|l|}{ Laboratory data } \\
\hline ESR $(\mathrm{mm} / \mathrm{hr})$ & $29.4 .2 \pm 28.0$ & $14.2 \pm 21.7$ & $9.6 \pm 8.4$ & & 0.070 \\
\hline $\mathrm{HbA1c}(\%)$ & $8.3 \pm 1.8$ & $5.9 \pm 2.5$ & $6.2 \pm 0.6$ & & $0.003^{*}$ \\
\hline Recurrence, $\mathrm{N}$ of patients (\%) & $1(4.0)$ & $2(8.0)$ & $1(33.3)$ & & 0.190 \\
\hline Duration of course (month) & $2.0 \pm 1.0$ & $1.6 \pm 0.8$ & $0.9 \pm 0.4$ & $1.7 \pm 0.9$ & $0.044^{*}$ \\
\hline Duration of follow-up (year) & $3.4 \pm 3.1$ & $4.9 \pm 3.6$ & $3.6 \pm 0.3$ & $4.0 \pm 3.3$ & \\
\hline Total recovery, N (\%) & $24(92.3)$ & $26(96.3)$ & $5(100.0)$ & $55(94.8)$ & 0.695 \\
\hline
\end{tabular}

Symbols and abbreviations: $N$ number; $C N$ cranial nerve; ESR elevated sedimentation rate; HbA1c glycohemoglobin. Footnotes: ${ }^{*}=$ statistically significant value.

partial patients with ocular diabetic neuropathy $(63.6 \%)$ did so $(\mathrm{p}=0.040$, Table 2$)$.

\section{Discussion}

The current study has several findings. Benign THS and ocular diabetic neuropathy accounted for the majority of cases of painful ophthalmoplegia with normal cranial imaging (91.4\%). Clinical presentations were similar between the groups, except for the age of onset, response to glucocorticoids, and duration of disease course. Nearly all patients with benign THS and OM had an immediate improvement in ocular motor function after initiation of

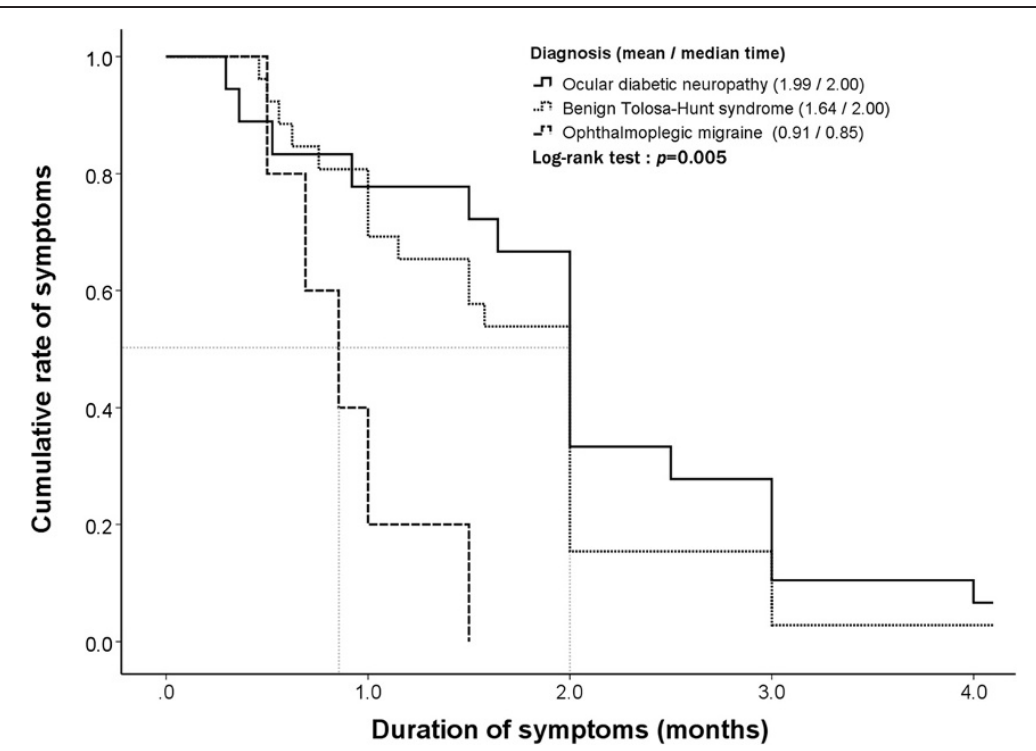

Figure 2 The clinical courses of ocular diabetic neuropathy, benign Tolosa-Hunt syndrome (THS), and ophthalmoplegic migraine (OM) are illustrated. The primary study endpoint was the time to complete resolution of painful ophthalmoplegia. The duration of clinical course differed among these groups (Log-rank test, $p=0.005$ ). OM (mean $=0.91$ months) showed a significantly more rapid recovery than benign THS (mean $=1.64$ months) and ocular diabetic neuropathy (mean $=1.99$ months). Overall, most of the patients recovered completely $(94.8 \%)$. 
Table 2 Response rate of pain and diplopia to glucocorticoids in cases of painful ophthalmoplegia with normal neuroimaging

\begin{tabular}{|c|c|c|c|c|c|}
\hline Response to glucocorticoids & Ocular diabetic neuropathy & Benign Tolosa-Hunt syndrome & Ophthalmoplegic migraine & Total & $p$ value \\
\hline Pain, N (\%) & $10(90.9)$ & $20(100.0)$ & $4(100.0)$ & $34(97.1)$ & 0.325 \\
\hline Diplopia, N (\%) & $7(63.6)$ & $19(95.0)$ & $4(100.0)$ & $30(85.7)$ & 0.040 \\
\hline
\end{tabular}

A positive response to glucocorticoids was defined as relief of symptoms or improvement of neurologic signs within 3 days of glucocorticoid treatment. $\mathrm{MRI}=$ magnetic resonance imaging; $\mathrm{N}=$ number

glucocorticoid treatment, whereas only partial patients with diabetic ophthalmoplegia did so. Patients with OM recovered more rapidly than the other groups did. Overall, most patients recovered completely during the follow-up period.

Patients with ocular diabetic neuropathy were significantly older than patients with benign THS and OM. This result is in accordance with previous reports; the mean reported age of diabetic ophthalmoplegias ranges from 50.2 to 68 years [6,17-19], which is more than that of benign THS (48.6 years) and adult OM (36.4 years) $[8,9]$. Diabetic neuropathy is predominantly a disease of older adults [20], whereas $\mathrm{OM}$ predominantly affects children and younger adults $[7,8]$, and THS may present at any age $[1,9]$.

Glucocorticoid administration is of therapeutic and partial diagnostic utility. The response of diplopia resolving was of higher diagnostic value than that of pain relieving. Orbital pain was dramatically relieved after glucocorticoid treatment in almost all patients. Regarding diplopia resolving, only THS and OM respond exquisitely to glucocorticoid therapy [1,7] The immediate improvement of ocular motor nerve dysfunction might reflect the inflammatory pathogenesis of these diseases $[7,12,21,22]$. In contrast, ischemic pathogenesis associated with ocular diabetic neuropathy $[5,23,24]$ decreases the response to glucocorticoids, even though glucocorticoids can partially relieve pain caused by ischemic injury.

Patients with OM recovered faster than patients with THS and ocular diabetic neuropathy, consistent with previously reported results $[7,10,18,19,25,26]$. The average recovery time of $\mathrm{OM}$ is approximately 3 weeks, whereas that of THS is approximately 2 months and ocular diabetic neuropathy is $3-4$ months $[7,9,10,18,19]$. Although the disease course varied among groups, the outcomes were generally good. In our experience, most patients recovered completely within 3 months. Physicians should be more vigilant if a patient fails to improve within 3 months, and further aggressive investigation may be indicated.

Pupil sparing was not only common in patients with ocular diabetic neuropathy, but was also common in patients with THS and OM. It is widely accepted that pupillary dysfunction is suggestive of aneurysmal or neoplastic compressive lesions [27,28], and it is not common in diabetic ophthalmoplegia [25,26,29]. Only $14-18 \%$ of patients with diabetic ophthalmoplegia develop pupillary dysfunction [29]. In addition, the pupil is occasionally affected in THS [4]. Although a majority of child patients with OM have pupillary involvement [7], adult patients with OMs tend to spare pupillary response [8]. Pupil sparing can be used as a potential marker for differentiating these ophthalmoplegias from those caused by structural compressive lesions.

Occasional reports have documented an elevated ESR in the acute stage of THS [1]. However, there is no convincing evidence for a correlation between connective tissue disease and THS. In our study, the ESR results were grossly normal in patients with benign THS. Therefore, ESR is of no diagnostic value in differentiating THS from other causes.

In the past, the absence of radiographic evidence of inflammation in patients with THS was confusing to clinicians, and a diagnosis of "idiopathic painful ophthalmoplegia" was applied to these patients [30-32]. The eponym of "benign THS" was first introduced by La Mantia et al. in 2006 to describe these patients [9]. According to the review by La Mantia et al., half of the THS patients manifest as benign condition. Given the nosography of benign THS has been proposed for less than a decade, it is probable that such cases will continue to be diagnosed. The exact proportion of benign and inflammatory cases remains to be determined. A strong similarity between benign and inflammatory THS was recently identified, and inflammation may contribute to the pathogenesis of both conditions [10]. An official definition and elaboration of benign THS is essential for clinical practice.

Painful ophthalmoplegia usually mandates an extensive systemic workup for an underlying neoplastic, inflammatory, infective or autoimmune disease. Because of the limitations of a retrospective study, an exhaustive checkup for screening was not possible. However, all recruited patients had ancillary laboratory workup, cranial MR imaging, and cerebral vascular investigation. In addition, none of the patients had newly diagnosed malignancies, autoimmune disease, or CNS infection during the follow-up period. Furthermore, the results were generally favorable during the follow-up period and all patients completely recovered or experienced minor sequelae. Therefore, after the follow-up, we could conclude that other causes of painful ophthalmoplegia were less likely and further confirmed the suitability of our diagnoses.

In this study, the classification was based on the ICHD-2 criteria and clinical specificity of each disease. Patients with definite diabetes (fasting plasma glucose $\geq$ $126 \mathrm{mg} / \mathrm{dL}$ or $\mathrm{HbA} 1 \mathrm{c} \geq 6.5 \%$ ) were designated as ocular 
diabetic neuropathy [11]. In fact, it is difficult to differentiate ocular diabetic neuropathy from benign THS comorbid with diabetes or impaired glucose tolerance at this present technological stage. The limitation of the diagnostic criteria might lead to selection bias in the classification.

Diabetic neuropathy may manifest before the diagnosis of DM [5]. It is possible that patients manifesting ophthalmoplegia with undiagnosed DM could be mistaken as those with THS. However, diabetic cranial neuropathies mainly occur in older individuals with a long duration of diabetes (mean $=8.5 \sim 16$ years) $[6,17,19]$; an occult diagnosis of diabetes is very less likely at this stage. In addition, none of the THS or OM patients in this study had a diagnosis of diabetes mellitus (fasting plasma glucose $\geq 126 \mathrm{mg} / \mathrm{dL}$ or $\mathrm{HbA} 1 \mathrm{c} \geq 6.5 \%$ ) or impaired fasting glucose (fasting plasma glucose $\geq 100 \mathrm{mg} / \mathrm{dL}$ ) [11]. Due to the limitation of a retrospective study, the data of impaired glucose tolerance (IGT), tested by oral glucose tolerance test, were incomplete in THS patients. Further prospective studies are warranted to clarify the relationship of benign THS and glucose intolerance.

Our study also had other limitations. First, the sample size of each group, especially the OM group, might be too small for adequate comparisons. Second, the conclusion that steroid is of partial diagnostic utility might result from the selection bias caused by the diagnostic criteria. Third, the retrospective analysis limited our ability to collect detailed information, forcing a reliance on documented findings of examinations. Fourth, histological examinations for pathological diagnoses lacked. Nevertheless, this study provides useful information about the clinical features of painful ophthalmoplegia and may warrant largescale prospective studies to assess the optimal treatment approach.

\section{Conclusions}

In summary, ocular diabetic neuropathy and benign THS account for most of the painful ophthalmoplegias in patients with normal cranial imaging. Glucocorticoid administration is of therapeutic and partial diagnostic utility because THS and OM respond exquisitely to the treatment. Patients with OM recover more rapidly than the other groups do. Patient outcomes are generally good.

\section{Consent}

The Institutional Review Boards of the Chang Gung Memorial Hospital waived the need for individual informed consent because all data were anonymized and de-identified prior to analysis.

\section{Competing interests}

The authors declare that there is no conflict of interests.

\section{Authors' contributions}

$\mathrm{CH}$ : acquisition, analysis and interpretation of data, drafting the manuscript. KC: analysis and interpretation of data. CC, ML, HC, RL and CL: substantial contributions to conception and design. YW and YC: reviewing and evaluating cranial images. HT: interpretation of data. LR: revising manuscript critically for important intellectual content. All authors read and approved the final manuscript.

\section{Acknowledgements}

We thank all the patients who participated in this study.

\section{Author details}

${ }^{1}$ Department of Neurology, Kaohsiung Municipal Hsiaokang Hospital, Kaohsiung, Taiwan. ${ }^{2}$ Department of Neurology, Kaohsiung Medical University Chung-Ho Memorial Hospital; Kaohsiung Medical University, Kaohsiung, Taiwan. ${ }^{3}$ Department of Neurology, Linkou Campus, Chang Gung Memorial Hospital and University College of Medicine, Gueishan Township, Taiwan. ${ }^{4}$ Department of Radiology, Linkou Campus, Chang Gung Memorial Hospital and University College of Medicine, Gueishan Township, Taiwan.

${ }^{5}$ Department of Radiology, Keelung campus, Chang Gung Memorial Hospital and University College of Medicine, Keelung City, Taiwan. ${ }^{6}$ Biostatistical Center for Clinical Research, Chang Gung Memorial Hospital, Gueishan Township, Taiwan.

Received: 16 July 2013 Accepted: 7 January 2014

Published: 9 January 2014

\section{Reference}

1. Kline LB, Hoyt WF: The Tolosa-Hunt syndrome. J Neurol Neurosurg Psychiatry 2001, 71(5):577-582.

2. Wu HC, Ro LS, Chen CJ, Chen ST, Lee TH, Chen YC, Chen CM: Isolated ocular motor nerve palsy in dural carotid-cavernous sinus fistula. Eur J Neurol 2006, 13(11):1221-1225.

3. Huston J 3rd, Nichols DA, Luetmer PH, Goodwin JT, Meyer FB, Wiebers DO, Weaver AL: Blinded prospective evaluation of sensitivity of MR angiography to known intracranial aneurysms: importance of aneurysm size. AJNR Am J Neuroradiol 1994, 15(9):1607-1614.

4. Headache Classification Subcommittee of the International Headache S: The international classification of headache disorders. Cephalalgia 2004, 24(1):9-160

5. Boulton AJ, Malik RA, Arezzo JC, Sosenko JM: Diabetic somatic neuropathies. Diabetes Care 2004, 27(6):1458-1486

6. Watanabe K, Hagura R, Akanuma Y, Takasu T, Kajinuma H, Kuzuya N, Irie M: Characteristics of cranial nerve palsies in diabetic patients. Diabetes Res Clin Pract 1990, 10(1):19-27.

7. McMillan HJ, Keene DL, Jacob P, Humphreys P: Ophthalmoplegic migraine: inflammatory neuropathy with secondary migraine? Can J Neurol Sci 2007, 34(3):349-355.

8. Lal V, Sahota P, Singh P, Gupta A, Prabhakar S: Ophthalmoplegia with migraine in adults: is it ophthalmoplegic migraine? Headache 2009, 49(6):838-850.

9. La Mantia L, Curone M, Rapoport AM, Bussone G, International Headache S: Tolosa-Hunt syndrome: critical literature review based on IHS 2004 criteria. Cephalalgia 2006, 26(7):772-781.

10. Hung $\mathrm{CH}$, Chang KH, Wu YM, Chen YL, Lyu RK, Chang HS, Wu YR, Chen CM, Huang CC, Chu CC, et al: A comparison of benign and inflammatory manifestations of Tolosa-Hunt syndrome. Cephalalgia 2013, 33(10):842-852.

11. American Diabetes A: Standards of medical care in diabetes-2011. Diabetes Care 2011, 34(Suppl 1):S11-S61.

12. Hunt WE, Meagher JN, Lefever HE, Zeman W: Painful opthalmoplegia. Its relation to indolent inflammation of the carvernous sinus. Neurology 1961, 11:56-62.

13. Cakirer S: MRI findings in Tolosa-Hunt syndrome before and after systemic corticosteroid therapy. Eur J Radio/ 2003, 45(2):83-90.

14. Giraud P, Valade D, Lanteri-Minet M, Donnet A, Geraud G, Guegan-Massardier E: Is migraine with cranial nerve palsy an ophthalmoplegic migraine? $J$ Headache Pain 2007, 8(2):119-122.

15. Smith JL, Taxdal DS: Painful ophthalmoplegia. The Tolosa-Hunt syndrome. Am J Ophthalmol 1966, 61(6):1466-1472.

16. Colnaghi S, Versino M, Marchioni E, Pichiecchio A, Bastianello S, Cosi V, Nappi G: ICHD-II diagnostic criteria for Tolosa-Hunt syndrome in 
idiopathic inflammatory syndromes of the orbit and/or the cavernous sinus. Cephalalgia 2008, 28(6):577-584.

17. Greco D, Gambina F, Maggio F: Ophthalmoplegia in diabetes mellitus: a retrospective study. Acta Diabetol 2009, 46(1):23-26.

18. Jacobson DM: Pupil involvement in patients with diabetes-associated oculomotor nerve palsy. Arch Ophthalmol 1998, 116(6):723-727.

19. Acaroglu G, Akinci A, Zilelioglu O: Retinopathy in patients with diabetic ophthalmoplegia. Ophthalmologica 2008, 222(4):225-228.

20. Aaberg ML, Burch DM, Hud ZR, Zacharias MP: Gender differences in the onset of diabetic neuropathy. J Diabetes Complications 2008, 22(2):83-87.

21. Tolosa E: Periarteritic lesions of the carotid siphon with the clinical features of a carotid infraclinoidal aneurysm. I Neurol Neurosurg Psychiatry 1954, 17(4):300-302.

22. Carlow TJ: Oculomotor ophthalmoplegic migraine: is it really migraine? I Neuroophthalmol 2002, 22(3):215-221.

23. Asbury AK, Aldredge H, Hershberg R, Fisher CM: Oculomotor palsy in diabetes mellitus: a clinico-pathological study. Brain 1970, 93(3):555-566.

24. Smith BE, Dyck PJ: Subclinical histopathological changes in the oculomotor nerve in diabetes mellitus. Ann Neurol 1992, 32(3):376-385.

25. Tesfaye $\mathrm{S}$ : Diabetic neuropathy: achieving best practice. $\mathrm{Br} J$ Diabetes VasC Dis 2003, 3(2):112-117.

26. Leslie $D$, Ellis C: Clinical course following diabetic ocular palsy. Postgrad Med J 1978, 54(638):791-792.

27. Kissel JT, Burde RM, Klingele TG, Zeiger HE: Pupil-sparing oculomotor palsies with internal carotid-posterior communicating artery aneurysms. Ann Neurol 1983, 13(2):149-154.

28. Keane JR: Aneurysms and third nerve palsies. Ann Neurol 1983, 14(6):696-697.

29. Goldstein JE, Cogan DG: Diabetic ophthalmoplegia with special reference to the pupil. Arch Ophthalmol 1960, 64:592-600

30. Nieri A, Bazan R, Almeida L, Rocha FC, Raffin CN, Bigal ME, Schelp AO: Bilateral painful idiopathic ophthalmoplegia: a case report. Headache 2007, 47(6):848-851.

31. Berthier E, Vighetto A, Aimard G, Confavreux C, Trillet M: Idiopathic cranial polyneuropathies. Rev Neurol 1992, 148(1):20-23.

32. Foerderreuther $\mathrm{S}$, von Maydell $\mathrm{R}$, Straube A: A CPH-like picture in two patients with an orbitocavernous sinus syndrome. Cephalalgia 1997, 17(5):608-611.

doi:10.1186/1471-2377-14-7

Cite this article as: Hung et al:: Painful ophthalmoplegia with normal cranial imaging. BMC Neurology 2014 14:7.

\section{Submit your next manuscript to BioMed Central and take full advantage of:}

- Convenient online submission

- Thorough peer review

- No space constraints or color figure charges

- Immediate publication on acceptance

- Inclusion in PubMed, CAS, Scopus and Google Scholar

- Research which is freely available for redistribution 